\title{
Interactive comment on "Fatty acid carbon isotopes: a new indicator of marine Antarctic paleoproductivity?" by Kate Ashley et al.
}

\section{Anonymous Referee \#3}

Received and published: 3 July 2020

The manuscript by Ashley et al. proposes the use of the carbon isotope composition of selected fatty acids present in sediments as a palaeoproductivity proxy in an Antarctic polynya environment (Adélie Land). The topic, totally in line with the journal Biogeosciences, is worth being investigated as proxys of paleoproductivity, especially in Polar Regions, are still lacking. The authors present an interesting set of quantitative and isotopic data, and based on their expertise in polar environments, discuss their possible significance in terms of biogeochemical changes recorded in sediments. The approach is interesting but the discussion and the conclusions raised by the authors may appear a little over-optimistic as many assumptions are made and some potential biases are discarded too easily and/or overlooked. There are a number of issues that the authors should take into consideration before the manuscript can be considered for

Printer-friendly version

Discussion paper 
publication. Comments are made chronologically, regardless of their importance.

Line 89 and manuscript throughout: It should be made clear in the manuscript that the data are based solely on free FA which represent only part of the total FA present in sediments (especially in modern to sub-recent sediments). If the selected FA indeed represent tracers of primary production, than it would be worth having a look at the bound (esterified) FA as well.

Lines 90-91: Please give more details on the use of $\mathrm{BF} / \mathrm{MeOH}$ as this treatment is known to be deleterious for some (poly)unsaturated FA.

Lines 91-94 and Fig. S2. Please give more detail on the chromatographic conditions used (for both GC and GC-MS analyses) and refer to figure S2. Also, the quality of the GC trace shown in figure S2 must be improved as, at such, a clear absence of unsaturated FA (which elute very close to saturated FA) is difficult to admit. As the authors know, the quality of compound specific $13 \mathrm{C}$ analyses is highly dependent on the purity of the compounds investigated and the absence of co-elution. Unsaturated FA often exhibit distinct $13 \mathrm{C}$ compositions compared to their saturated counterparts, so even small co-elution may significantly bias ïĄd'13C values of saturated FA. An additional purification step using $\mathrm{Si} / \mathrm{Ag}+$ column chromatography may have been worth being investigated. Lastly, the peak attributed to the internal standard (C19 alkane) in Fig S2 is in fact most probably the C14 FA as it is not possible that the C19 FA elutes 15 minutes later than the $\mathrm{C} 19$ alkane. Please check peaks assignment (including the IS).

Lines 94-97. In line with the previous comment, more detail is undoubtedly required concerning CSIA. Which type of GC and conditions were used including the characteristics of the capillary column, the temperature of the interface and the oven, etc? Does 'Duplicate measures' means that each sample was analyzed twice? If so, the error given is a min-max and not a standard deviation. Were the measured ïAd' $13 \mathrm{C}$ values corrected 1) for the methyl group added through derivatization and 2) for instrument

Printer-friendly version

Discussion paper 
deviation using a standard mixture? Are the stable isotope ratios expressed relative to the standard Vienna Pee Dee Belemnite (V-PDB)?

Line 102: which IS were used for $\mathrm{HBI}$ ?

Line 106: This is unclear as it sounds like a repetition of the previous sentence.

Lines 125-126: The sole presence of saturated FA in (sub)actual sediments of (hyper)productive areas is very unusual (this is an additional reason why a very clear GC trace is needed in Fig. S2 which could even be included in the main manuscript). Would it be possible that unsaturated FA were (partly) destroyed by the BF3 treatment?

Line 132: The actual figure 4 should become figure 2 and, consequently, actual figures 2 and 3 should become figures 3 and 4 , respectively.

Actual figure 4: The upward displacement of either one or two GC trace(s) within each group would make the different trends easier to compare. The horizontal axis could also be homogenized with that of figures 5 and 6 (age or eventually both depth and age, and from right to left).

Line 144 and all along the manuscript: Please also give an estimated time span when speaking in $\mathrm{cm}$ depth.

Lines 166-168: In Dalsgaard et al., the mean proportion of C18:0 FA in Prymnesiophyceae is only $3 \%$ ! Please specify it.

Line 170: 'higher preservation rate' may be misleading; replace with 'higher potential of preservation'.

Line 170: replace 'its proportion' with 'its relative proportion'.

Lines 166-181: This whole section deals with proportions of C18 FA in laboratory cultures which can show great differences with the environment. Could authors comment on this? 
Lines 181-183 and more generally: This is one of my main concerns. The C18 FA can be produced by various type of (micro)organisms and assigning a single origin to this compound is rather daring. Authors should definitely support their hypothesis and comment about other potential sources of this compound such as bacteria, macrofauna, zooplankton, atmospheric inputs, land plants ... One would also expect concentration profiles to be combined with $\mathrm{d} 13 \mathrm{C}$ values to strengthen interpretations on the origin of individual biomarkers.

Line 184 and thereafter: The same comment (as that made for the C18 FA) holds for the C24 FA. In this case isotopic data could be additionally used to support a planktonic (vs terrestrial plants) origin.

Line 200 and thereafter: This is true but the degradation rates of lipid biomarkers appear strongly dependent on the redox conditions. Could authors give information on the redox state at the water-sediment interface and the possible influence of bioturbation in the surficial sediments?

Lines 227-229: Could this be due to an impact of bioturbation and/or to microbial production within the sediment?

Lines 257-259: A similarity between the concentration profiles of C18 FA, HBI triene and $\mathrm{HBI}$ diene is not obvious in figure 5 . Authors are encouraged to reconsider/specify those words.

Lines 261-262: This sentence is not clear. Do authors mean: '.... and to diagenetic transformation within the sediments including sulfurisation (ref), isomerisation (ref) and cyclisation (ref) reactions'?

Lines 262-264: This statement is misleading and in contradiction with section 3.4. Clearly, one cannot speak about a better preservation in the top sediments. The concentrations of $\mathrm{HBI}$ reflect the flux of lipids reaching the seafloor while the decrease in concentration downcore reflects enhanced degradation in the first $\mathrm{cm}$ of sediments (yet

Printer-friendly version

Discussion paper
Interactive comment

C4 
possibly including variations in productivity).

Lines 272-273: I agree but this holds true if diagenetic conditions remain the same through time. Any indication on potential variations in the redox state of the water column and water-sediment interface back in time?

Line 290: Again the concept that preservation of organic matter is better in surficial (younger) sediments is unfounded and in contradiction with section 3.4. It should be revised throughout the whole manuscript.

Lines 311-314 and thereafter: I don't think such a difference can be considered really significant (keeping in mind that the reproducibility was $+/-0.26$ per mill). This statement might be a little far-fetched and I would suggest to remove it.

Lines 355-356. I am not convinced by this statement when looking at the d13C profile of the C24 FA which shows a clear trend towards lighter values (2-3 per mill) within the last 150 years. Authors are mentioning this trend later on (lines 375-376). Could this be linked to increased land plant inputs due to ice retreat?

Lines 366-367: please temper with 'do not tend to produce high proportions of this compound'.

Lines 372-378: This again is somewhat speculative. If both FA have distinct origins, than the diagenetic impact on their 13C composition may be significantly different. What about the possibility that either one or both FA are being produced in the top sediments?

Section 4.3 (lines 522-530) and conclusions (lines 540-541): As considered for FA in actual figures 2 and 3 , a correlation table would help in highlighting putative relationships between lipid biomarker (concentration or $\mathrm{d} 13 \mathrm{C}$ ) profiles and specific phytoplanktonic species.

Printer-friendly version

Discussion paper

Interactive comment on Biogeosciences Discuss., https://doi.org/10.5194/bg-2020-124, 2020. 